\title{
Application of AI in Home Automation
}

\author{
Sandeep Kumar and Mohammed Abdul Qadeer
}

\begin{abstract}
Home automation is on horizon. It is an emerging technology and also a need of today. From the last decade a number of standards have been defined for home appliances. The main objectives of home automation are controlling, management and co-ordination of home appliances in a comfortable, effective and secure way.

On the other hand, Artificial Intelligence is evolving as a technology for developing automatic systems that can perceive the environment, learn from environment, and can make decision using case based reasoning. Using Vision capability, knowledge based, learn ability, decision making and reasoning the AI provides a better solution for almost all automatic systems.

In this paper we will see the types of home automation systems and then see how these system can utilize the AI tools so as to increase the effectiveness, powerfulness etc.
\end{abstract}

Index Terms-Home automation, artificial intelligence, knowledge base systems, secure home.

\section{INTRODUCTION}

One definition of an automated and networked home is "An automated and networked home is one in which every appliance can be remotely managed [1] from anywhere on the Internet with a simple Web browser" [2]- [5]. The general goal of the automatic-home movement is to use networking technology to integrate the devices, appliances and services found in homes so that the entire domestic living space can be controlled centrally or remotely [6].

Home wiring, the advance home developers are installing, typically adds several thousand dollars to the cost of a new home, and it is usually Ethernet or coaxial cable -- or some combination of both -- with other technologies in the mix. The network is being designed to make possible remote operation of appliances connected to the network.

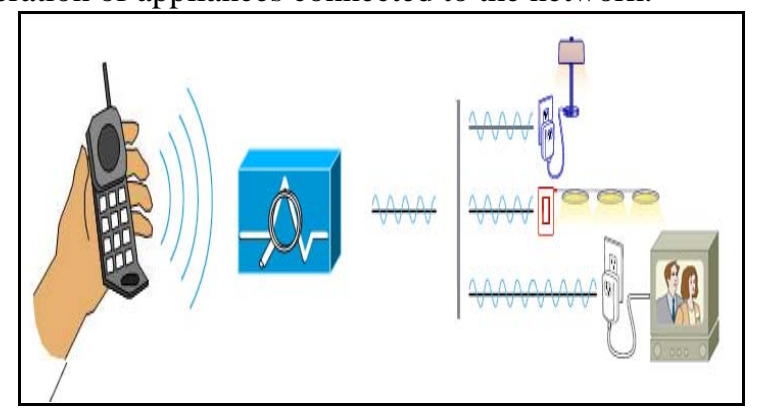

Fig. 1. Already available extra wiring for device controlling in smart infrastructure.

Manuscript received June 22, 2012; revised July 29, 2012. This work was supported in part by the Aligarh Muslim University.

Sandeep Kumar is with the Tata Consultancy Services Limited, Pune India (e-mail: sandeepkumar@zhcet.ac.in).

Mohammed Abdul Qadeer is with the Department of Computer Engg., Aligarh Muslim University. Uttar Pradesh, Aligarh, India (e-mail: maqadeer@gmail.com).

\section{A. Services Provided by the Home Automation System}

Comfortab A home automation system provides a large number of services which can broadly classified into following four categories:

1) le management of appliances

2) Remote controlling of appliances

3) Efficient utilization of home resources

4) Enhancing home security

Comfortable management includes automatic adjustment of AC(air conditioning) setting, fan regulation setting etc. Remote controlling services include accessing devices from remote location and setting them ON/OFF. Efficient utilization includes running the home appliances at their optimal setting (setting at which we get the required output at minimum cost). Last category of service includes all those services which are used for securing the home environment.

In this paper we will see the implementation of all these categories of services one by one and will see the application of AI techniques in implementation.

In Section-II we will see AI techniques \& Knowledge based system very briefly. Section-III covers the issues concerned with home automation. In Section-IV, V, VI and VII we have discussed the detailed implementation of systems for implementing the home automation system developed for services listed here.

\section{ARTIFiCial INTELLIGENCE AND KNOWLEDGe BASED SYSTEMS (KBS)}

$\mathrm{AI}$ is the collection of powerful and rigorous programming techniques studying the nature of intelligence by building computer systems, and the application of these concepts in solving real-world problems [7]. The growth in the areas of AI has been increased significantly from the last decade. There exist a number of AI tools that make an automation system more sophisticated but here we will discuss the knowledge based systems only as it is used frequently.

Knowledge Based System:

A knowledge-based system (KBS) [8] is an AI based system that contains a significant amount of knowledge in an explicit, declarative form. The area of KBS development has matured over the past two decades. It started with first-generation expert systems with a single flat knowledge base and a general reasoning engine, typically built in a rapid-prototyping fashion. This has now been replaced by methodological approaches that have many similarities with general software engineering practice. KBS development is best seen as software engineering for a particular class of application problems. These applications problems typically require some form of reasoning to produce the required results. In current business practice there is an increasing need for such systems, due to progression of information 
technology in our daily work. For home automation knowledge based systems can provide the base to store the user preferences and managing the home appliances accordingly.

\section{PRELIMINARIES}

If we talk home automation, there are following three main issues:

1) How to connect home appliances and apparatus

2) How to make two home appliances to communicate to each other

3) How to control and manage home appliances

As far as first issue is concerned, a number of standards have been developed for interconnecting the home devices and apparatus in a network so as to make their management much easier and comfortable. Summarized from [9]-[14] a following are the main networking technologies used for connecting devices in home environment:

1) Direct cable connection

2) Bluetooth Connection

3) Phone Line

4) Ethernet

5) Radio (Free) Network

6) AC Network

So we can connect the entire home devices by selecting any of the above mentioned network technologies.

Apart from connecting devices, the second issue regarding the home automation is "how to make two devices communicate to each other". For handling this issue a number of standards have been developed. Summarized from [10], [15]-[20] there are following leading communication technologies in home environment:

1) UPnP (Universal Plug and Play) devices

2) X-10 based devices

3) Infrared devices

4) Bluetooth Devices

5) IP based devices

All these technologies are well matured and have well settled standards. But, in this paper we will skip it as this is not of our concern.

Now we move toward third issue i.e. "how to manage and control the home appliances" which is the issue we are concerned with. This issue can be handles in two ways. First, by using traditional digital and microprocessor based systems like discussed in [13], [17], [19]. Second method for this is by using the sophisticated processing of artificially intelligent agents.

If we consider the first choice, then it is somewhat more common but at the cost of time efficiency as well as feature limitations. On the other hand AI based technique provides more efficient and featured services like easy video and audio processing, easy reasoning etc.

In this paper we will concentrate on the third issued of home automation i.e. management of home appliances and particularly, AI based home appliance controlling and management.

\section{Managing Home ApPliances for COMfortability}

As discussed in Section-I, the first service that a user expect from the home automation system is the comfortable management of devices.

e.g. the regulator setting of AC depends upon the temperature of the room. As the temperature increases the AC regulation rating (hence cooling rate) also need to be increased.

Such class of services is simplest of all types' services and has the structure as shown in the Fig. 2.

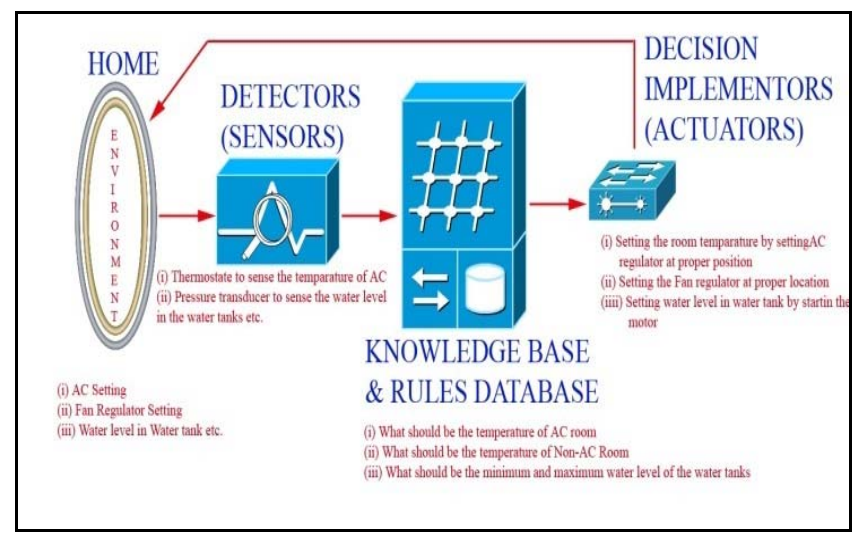

Fig. 2. Typical structure of home automation system designed for user comfortability.

Some features of such systems are:

1) These are closed loop systems

2) Sensors are transducers and other mechanism for sensing the current proximity condition e.g. sensing the room temperature

3) Actuators are simply the mechanism to change the environment according to the control signals received from the knowledge base.

4) Knowledge base $(\mathrm{KB})$ is the centralized part of the system and is the main part to discuss here.

This KB system can be implemented by using simple digital circuitry as discussed in [17] or using microprocessor systems as discussed in [11] but both suffer from the problem of manual setting. In both the system user has to decide and change the threshold setting whenever there is a change in the environment.

E.g. in summer AC is used to down the temperature while in winter the same are used to up the temperature. So, user has to change the setting when season changes because the system can't learn from its experience.

But using the KB system having learning capability, system can adjust the threshold setting as it gets the experience in its environment. For this system we need a KB system that can learn from the experience that "what is comfortable temperature" for the home users and can adjust the setting of AC and fan regulator accordingly.

\section{Controlling Home Appliances From Remote LOCATION}

The second feature provided by the home automation system is the remote access to devices and their management.

E.g. suppose you are going back to your home and it will take 20 minutes to reach your home. Now, you want to on 
you AC so that when you reach your home you find your home with comfortable temperature. In such a scenario you need to access your home appliances and also to control them from remote location.

The typical structure of home automation system for this type of services is given in Fig. 3.

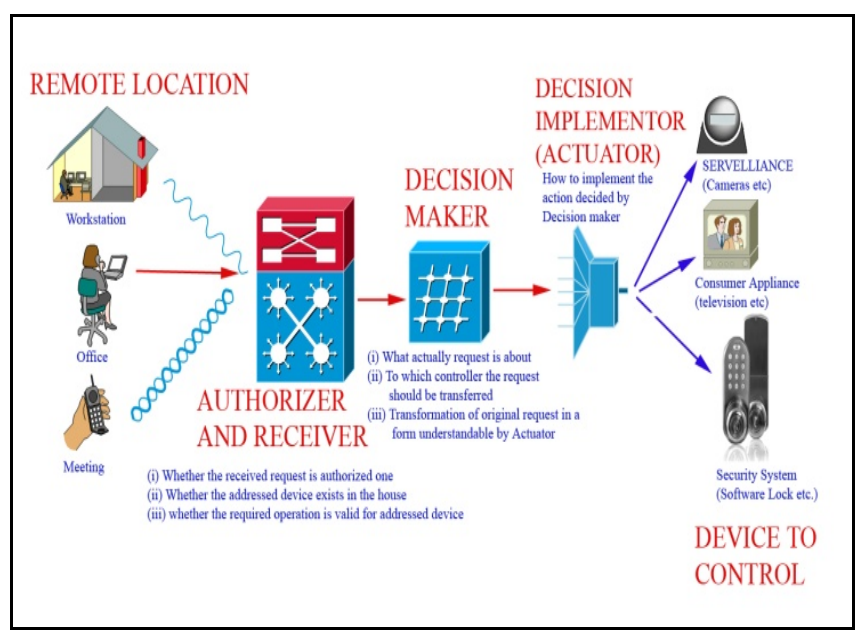

Fig. 3. Typical structure of home automation system designed for remote management of home devices

Following are main points to consider about this class of systems:

1) Authorizer and Receiver is an electronic system capable to receive the control signal. As discussed in Section-II there exist a number of such systems any of them can be used for this purpose. One important thing about this system is that it requires some authorization mechanism to ensure that the request is authorized one. For this purpose we can use some cryptographic techniques to encode and decode the request so that only authorized user can access the network.

2) The decision maker system is an AI based agent that can decide what action should be taken in response to received query.

E.g. suppose user just put the query that the room temperature should be $\mathrm{x}^{0} \mathrm{c}$. Now, this is the Decision Maker that will identify from its experience that the AC will maintain this temperature. And then it will determine setting of AC regulator corresponding to this particular temperature.

Of course, the same system can be implemented by some electronics circuitry but that will be more complex, less flexible and less featured as compared to this learning based (case based) AI agent system.

3) The third component of this system i.e. Actuator is similar to the action implementer in the previous system. So, we will not discuss anything about this here.

\section{EFFICIENT UTILIZATION OF HOME APPLIANCES}

This application of home automation system is not as common as discussed two applications. But, on the other hand if the automation system is applied in the industrial environment then it becomes the more prominent and beneficial feature of the automation system.
E.g. Suppose the AC of your office (or room) is ON but you are not in your room from last three hours. Then why the AC is still ON? This is just wastage of power. As said already, this power wastage is not much in case of home appliances but in industries power saving is one of the major cost cutting factor. So, for implementing this we need a system that can OFF the AC if it found that there is no person from the last significant time. We said here the word significant as the exact time can't be determined and depends upon the scenario (like for bed room the AC should down OFF if there is no person for one hour while in waiting room the $\mathrm{AC}$ should go down when there is no person from last two hours).

In such a scenario, we are supposed to have some automation system that can continuously pole the environment and can decide the appropriate action whenever needed. These systems should fast enough for responsiveness and effectiveness.

Fig. 4 shows the typical structure of the system that can provide this class of services. The working of the components of the system is as follows:

1) The status poller continuously senses the environment conditions and forwards the sensed condition-factors to analyzer and knowledge based database.

2) The Analyzer receives the current environment condition from poller and with the help of knowledge base database it analyses the variation in the environment. Now, depending upon the variation Analyzer gives the instruction to Actuator.

3) Again here the actuator is similar to the actuator discussed in previous sections.

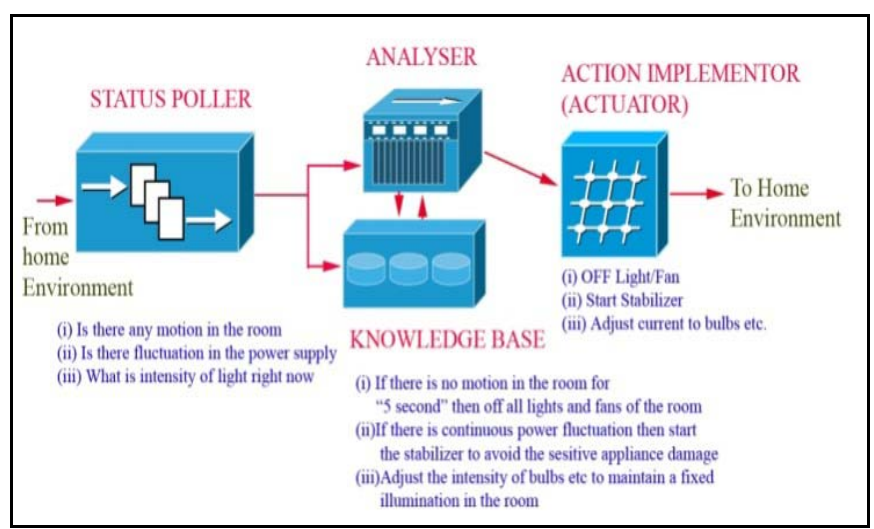

Fig. 4. Typical structure of home automation system designed for optimizing resource utilization.

The major differentiating factor of this system from previously discussed systems is that this is sequential while previous one was atomic, because, in this system the action to be taken depends upon the present and previous environment conditions.

\section{SECURE HOMES}

The last major application provided by the home automation system is to provide the home security. This is the most complex home automation system and the structure is given in the Fig. 5. 


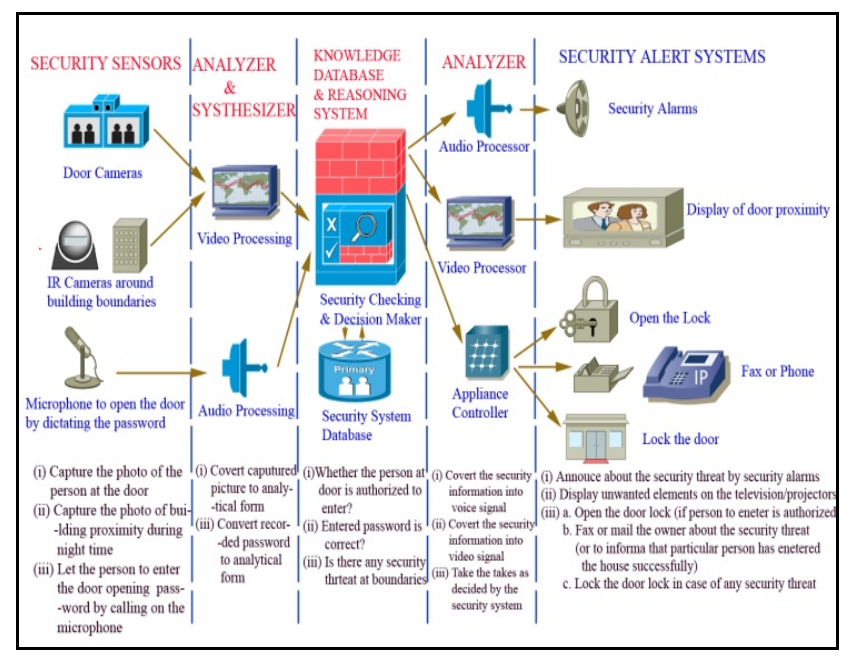

Fig. 5. Typical structure of AI based secure home.

The description of how system works is as follows:

1) Security sensors sense the environment for security threat.

2) The analyzer and synthesizer analyses the data received from the sensors and filter out any security threat. If it found any security threat, then it sends the sufficient information to reasoning system.

3) The Reasoning system apply the reasoning for detecting whether the security threat is really a threat, if yes then it fires the security alert system for alerting the user about this security threat by analyzer.

4) This analyzer is same as used at the second stage but opposite in nature. The previous converts the physical information into digital form while later one converts the digital information back to physical form to alert the user.

\section{ApPlication OF AI In Home AutOMAtion}

So in Section-IV, V, VI and VII we have discussed about the home automation taken one class of services at time. Now, in this Section we will concentrate on the application of AI in all the four forms of home automation system one by one.

\section{A. Use of AI in Comfortable Systems}

In these systems the application of AI is limited as most of the part can be easily implemented using some electronic circuitry. Here the only part where the AI is effective is the knowledge based database which should be learnable (as discussed previously) for system to be truly comfortable. Since AI tools are little bit costly it will increase the cost of the system but will make the system more comfortable, flexible, easy updatable etc.

\section{B. Use of AI in Remote Controlling Systems}

In these systems AI can be applied in the authorizer as well decision maker stage. Applying AI at the authorizer will increase the responsiveness and security and is more applicable when the environment under consideration is an industry where security is a major concern (e.g. Banks). On the other hand, Decision making part can utilize the case based reasoning of AI for effective and efficient management as it has to decide that which of the target device is corresponding to this particular instruction.

\section{Use of AI in Optimizing the Resource Performance}

In this system AI can used to implement knowledge base as discuss for previous systems and for Analyzer as it makes it more efficient in deciding the particular action. Moreover, if analyzer is learnable from its experience then it will make the system more optimized.

\section{Use of AI in Secure Systems}

The biggest use of AI is in these systems. Here we can apply following tools of AI for various applications:

1) Video Processing for security threat analysis

2) Image Processing for security threat analysis

3) Audio processing for security threat analysis

4) Knowledge base system for Security system database

5) Case based reasoning for analyzer and synthesizer

6) Decision Making in Security Checking and Decision making

So these systems use the AI exhaustibly. Presently, even though, different technologies are used for implementing these systems but in the near future AI will be the only implementation technique behind these systems as it is proved to be more sophisticated and effective tool for listed applications.

\section{CONCLUSION}

In this paper we started our discussion with home automation system by defining four major applications of these systems which are comfort ability, remote control, optimal resource utilization and security. After that we see the detailed structure of home automation for implementing these services one by one explaining the working of each system and use of heuristic based tools in these systems. At the last we discuss about the applications of AI tools in all four types of home automation systems.

From this discussion it is clear that $\mathrm{AI}$ is emerging as a very useful and applicable technology for Home automation. On the other hand, home automation systems provide AI a vast range of Application.

\section{REFERENCES}

[1] I. Han, H.-S. Park, Y.-K. Jeong, and K.-R. Park, “An integrated home server for communication, broadcast reception, and home automation,” IEEE Transactions on Consumer Electronics, vol. 52, no. 1, February 2006.

[2] P. M. Corcoran and J. Desbonnet, "Browser-style interfaces to a home automation network," IEEE Transactions on Consumer Electronics, vol. 43, no. 4, November 1997.

[3] A. Rosendahl, J. F. Hampe, and G. Botterweck, "Mobile home automation - merging mobile value added services and home automation technologies,” in Proc. Sixth International Conference on the Management of Mobile Business ICMB 2007.

[4] T. Uemukai, H. Hagino, T. Hara, M. Tsukamoto, K. Harumoto, and S. Nishio, "A WWW browsing method using a cellular phone in a remote display environment,” Data Processing Conference (99-HI-86,99-MBL-l 1), vo1. 99, no. 97, pp. 51-56, 1999.

[5] P. M. Cocoran, F. Papai, and A. Zoldi, "User interface technologies for home appliances and networks," IEEE Transactions on Consumer Electronics, vol. 44, no. 3.

[6] S. Openshaw and Ch. Openshaw, "Artificial intelligence in geography," Chichester, Wiley and Sons, 1997.

[7] P.-H. Speel, A. Th. Schreiber, W. van Joolingen, G. van Heijst, and G. J. Beijer, "Conceptual modelling for knowledge-based systems," Encyclopedia of Computer Science and Technology, Marce Dekker Inc., NewYork. 
[8] P. Klungboonkrong and M. A. P. Taylor. “A microcomputer-based system for multicriteria environmental impacts evaluation of urban road networks," Computer, Environment and Urban Systems, 1998, vol. 22, no. 5, pp. 425-446.

[9] P. Varshney, "Remote controlled home automation system," $M$ Tech 3rd Sem, ZHCET, AMU, 2006.

[10] G. Kannan and S. Vijayakumar, "Smart home tested for disabled people,” TIFAC CORE, Velammal Engineering College, Chennai, Mobile and Pervasive Computing-2008.

[11] P. Gwangro, "Trends of home network technologies and services," KRNET 2004, June, 2004.

[12] B. Rose, WJR Consulting Inc., "Home networks: A standards perspective,” IEEE Communication Magzine, December 2001

[13] H. R. Lee and J. W. Kim, "UPnP Protocol Extension for Contents Sharing among Digital Home Networks," KISS, vol. 3 1, no. 2, 2004.

[14] S. Knauth, R. Kistler, and Daniel, "Kaslin and alexander klapproth," UPnP Compression Implementation for Building Automation Devices

[15] Y. Zhang and M. G. Wu, "The study and development on intelligent lighting system based on X-10 protocol," China Illuminating Engineering Journal, vol. 15 no. 1, pp. 22-26, Mar. 2004.

[16] P. Varshney, N. Kumar, and M. Rihan, Implementation of Bluetooth Based Smart Home for Assisting the Physically Disabled.

[17] S. kumar, A. Gupta, H. Bhardwaj, and S. Islam, "Internet and PC based appliance control," ICICS 2009 Jordon.

[18] P. Kingery, "Digital X-10," Advanced Control Technologies .Inc, 2002.

[19] S. Kumar and M. A. Qadeer, "Universal device automation system (UDAS),"in Proc. ICCSIT 2009 China.

[20] Y. Lee' and J. W. Choi', "Remote-controlled home automation system via bluetooth home network," SICE Annual Conference in Fukui, Fukui University, Japan, August 4-6, 2003.

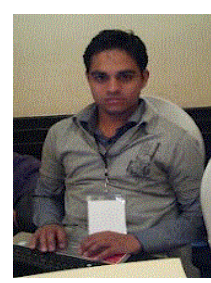

Sandeep Kumar is currently working with Microsoft on behalf of Tata Consultancy services limited as solution developer. From July 2012 he will be joining $\mathrm{PhD}$ program at IIT Mumbai. He has 15 publications in various conferences, journals and book chapters. He is associated with ACM, CSI, British Computer Society and other pioneering R\&D association.

His areas of interest include Artificial Intelligence, Neural Network and Application of computational techniques and programming in solving/analyzing biological problems/phenomenon.

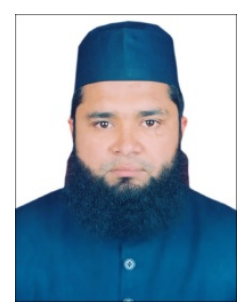

Mohammed A Qadeer is an Asst. Professor with the Department of Computer Engineering, Aligarh Muslim University, India. Earlier, he was working with Cisco Systems Inc. as a Network Consulting Engineer with the Advanced Services division in the APAC region. He received his B.Sc. Engineering (Computer Engineering) from Aligarh Muslim University in1996. He has an experience of 15 years in the area of computer networks and systems.

His areas of research are computer networks, wireless networks, mobile computing, next generation networks, IMS, LTE, WiMAX, 4G, WiBro etc. 